\title{
Effect of Psychological Intervention Combined with Family Cooperation on the Perioperative Quality of Life and Psychological States of Elderly Patients with Prostate Cancer Treated with Compound Kushen Injection
}

\author{
Jingyun Zhang, Caijian Li, Chengwei Fu, Jinkai Dong, Wei Guo, and Qianqian Zhu \\ Department of Urology, The Fifth Medical Centre, Chinese PLA General Hospital, Beijing 100071, China \\ Correspondence should be addressed to Qianqian Zhu; luketanp@163.com
}

Received 4 June 2021; Accepted 13 July 2021; Published 20 July 2021

Academic Editor: Alamgeer Yuchi

Copyright (C) 2021 Jingyun Zhang et al. This is an open access article distributed under the Creative Commons Attribution License, which permits unrestricted use, distribution, and reproduction in any medium, provided the original work is properly cited.

\begin{abstract}
Objective. The purpose of the study was to investigate the nursing effect of psychological intervention combined with family cooperation on elderly patients with prostate cancer treated with compound kushen injection and put forward effective suggestions. Methods. 122 elderly patients with prostate cancer admitted to our hospital from June 2018 to June 2019 were selected and randomly divided into a control group $(n=61)$ and experimental group $(n=61)$. The patients in the control group received routine nursing intervention during the perioperative period, while the patients in the experimental group were treated with psychological intervention combined with family cooperation on the basis of routine nursing. The quality of life and psychological states of patients in the two groups were statistically analyzed. Results. The evaluation of psychological states at 24 hours before surgery and 24 hours before discharge in the experimental group was significantly better than that in the control group $(P<0.05)$, with statistical significance. On comparing the basic conditions between the two groups in the perioperative period, the length of hospitalization, length of catheter retention after surgery, and incidence of complications in the experimental group were all significantly better than those in the control group $(P<0.05)$, with statistical significance. The satisfaction of patients with the nursing process in both groups was recorded and statistically analyzed through questionnaires. The satisfaction with nursing process in the experimental group was significantly higher than that in the control group $(P<0.05)$, with statistical significance. The quality of life of the patients was followed up at three months after discharge. The quality of life of the experimental group was significantly better than that of the control group $(P<0.05)$, with statistical significance. Conclusion. Psychological intervention combined with family cooperation for the elderly patients with prostate cancer treated with compound kushen injection is beneficial to improve their psychological states, encourage them to face the disease in a more positive manner, effectively improve the quality of life after intervention, ensure the therapeutic effect during perioperative period, increase happiness index, and enhance their satisfaction with the nursing process, which is worthy of clinical application and popularization.
\end{abstract}

\section{Introduction}

According to relevant reports, with the aggravation of aging, people's life concept and quality of life have changed greatly, and the incidence of prostate cancer increases year by year [1-3]. Many studies have shown that compound kushen injection has a certain synergistic effect as an anticancer treatment, relieving pain, enhancing immunity, and stopping bleeding. It is an effective adjuvant drug for clinical treatment of prostate cancer and can significantly improve the clinical benefit of patients. To further ensure good prognosis in elderly patients with prostate cancer, scientific nursing measures must be combined. In elderly patients, the social adjustment capacity is decreased, and they are shy of the arrival symptoms of this disease, which results in huge psychological burdens. Therefore, most elderly patients with prostate cancer face stressful psychological reactions in the perioperative period, including depression and anxiety, 
which further adversely affect the therapeutic efficacy and physical recovery [4-6]. Based on this, in this study, psychological intervention combined with family cooperation was introduced to elderly patients with prostate cancer treated with compound kushen injection, and the patients' life quality and psychological states were evaluated, so as to further analyze the intervention's effect and provide data support for clinical research. The results of the study are summarized as follows.

\section{Materials and Methods}

2.1. General Information. One hundred and twenty-two elderly patients with prostate cancer admitted to our hospital from June 2018 to June 2019 were selected and randomly divided into control group $(n=61)$ and experimental group $(n=61)$, aging from 55 to 81 years, with the average age of 69.37 years. There were no statistical differences in the comparison of general data such as age in both groups $(P>0.05)$, which was comparable, and the comparison of general data between the two groups is detailed in Table 1.

2.2. Inclusion Criteria. The inclusion criteria are as follows. (1) Patients met the clinical diagnostic criteria for prostate cancer according to the Diagnostic Criteria for Prostate Cancer. (2) All patients received compound kushen injection as an adjuvant therapy. (3) Patients had complete clinical records. (4) This study was approved by the Hospital Ethics Committee, and the patients and their families were informed of the purpose and process of this study and signed the informed consent.

2.3. Exclusion Criteria. The exclusion criteria are as follows. (1) Patients had other malignant and severe diseases. (2) Patients had other acute or long-term urinary system diseases. (3) Patients had cognitive impairment, such as mental disorders, or refused to cooperate with the study. (4) Patients had incomplete clinical data.

2.4. Methods. Patients in the control group were treated with the routine clinical nursing. According to the diagnosis results, preoperative examination and preparation were performed. The medical staff actively participated in the process of formulating the patients' surgical plans, reasonably evaluated patients' conditions, and made a scientific nursing intervention program. Besides, during the surgery, the medical staff cooperated with surgeon to complete the surgical process and paid close attention to the changes of patients' vital signs. After the surgery, routine index examination for patients was performed, and patients' dietary management and reasonable mix of nutrition were also taken into consideration [7-9].

Patients in the experimental group received psychological intervention combined with family cooperation. After patients' admission to hospital, psychological intervention was conducted on the basis of routine nursing by keeping an eye out for patient's psychological changes.
According to the patients' conditions, the medical staff informed patients and their family members of the cause of the disease, specific conditions, and treatment plan of prostate cancer in a gentle way. Additionally, the medical staff also explained the relevant knowledge of prostate cancer in detail, thoroughly understood the concerns of patients and their family members, and paid attention to emotional comfort. By describing similar successful cases, the medical staff increased the confidence of patients and their families and eliminated the fear before the surgery. After the surgery, patients and their families were informed of the results of the surgery in time and were encouraged to cooperate closely with the treatment. The patients' family members were told to conduct good supervision and support work to further increase the patients' confidence [10-12]. The nursing staff and family members cooperated with each other to perform the routine nursing and introduce psychological intervention during the perioperative period and observed the psychological changes of the patients acutely, so as to increase the patients' sense of security [13].

\subsection{Observation Indexes}

2.5.1. Scores of Self-Rating Anxiety Scale (SAS) and SelfRating Depression Scale (SDS) in Both Groups. Self-rating anxiety scale (SAS) and self-rating depression scale (SDS) were adopted to evaluate the psychological states of patients at 24 hours after admission, 24 hours before the surgery, and 24 hours before discharge, with the total score of 100 points, and higher scores indicated patients' severer psychological conditions.

2.5.2. Basic Conditions in Both Groups during the Perioperative Period. The basic conditions of the patients in the two groups during the perioperative period were recorded in detail, including the length of the operation, bladder irrigation time, length of catheter retention after surgery, length of hospitalization, and incidence of complications.

2.5.3. Nursing Satisfaction in Both Groups. The nursing satisfaction of patients in the two groups was investigated with a self-made questionnaire from the hospital, which mainly included the nurses' attitudes, quality of work, professional degree, and so on, with the total score of 100 points. A score above 90 points indicated "very satisfied," a score of 70-90 points indicated "basically satisfied," and a score below 70 points indicated "unsatisfied." Total nursing satisfaction $=$ basically satisfied + very satisfied .

2.5.4. Statistical Analysis of the Postoperative Quality of Life in Both Groups. The quality of life of the patients after surgery was evaluated by three evaluation indexes, international prostate symptom score, rehabilitation knowledge evaluation, and comprehensive evaluation of quality of life, so as to further record and analyze the patients' physical recovery, family emotion environment, body function, social skill and psychological states. 
TABLE 1: General information of elderly patients with prostate cancer in both groups $(n=61)$.

\begin{tabular}{|c|c|c|c|c|}
\hline & Experimental group & Control group & $T$ or $X^{2}$ & $P$ \\
\hline Age (years) & $62.4 \pm 5.63$ & $63.6 \pm 5.62$ & 1.1781 & 0.2411 \\
\hline $\begin{array}{l}\text { Education } \\
\text { Below elementary education } \\
\text { Elementary education and above }\end{array}$ & $\begin{array}{l}20(32.79 \%) \\
41(67.21 \%)\end{array}$ & $\begin{array}{l}18(29.51 \%) \\
43(70.49 \%)\end{array}$ & 0.1529 & 0.696 \\
\hline $\begin{array}{l}\text { Past medical history } \\
\text { Hypertension } \\
\text { Diabetes } \\
\text { None }\end{array}$ & $\begin{array}{l}16(26.23 \%) \\
12(19.67 \%) \\
33(54.09 \%)\end{array}$ & $\begin{array}{ll}14 & (22.95 \%) \\
13 & (21.31 \%) \\
34 & (55.74 \%)\end{array}$ & 0.0331 & 0.856 \\
\hline $\begin{array}{l}\text { Smoking } \\
\text { Yes } \\
\text { No } \\
\end{array}$ & $\begin{array}{l}50(81.97 \%) \\
11(18.03 \%)\end{array}$ & $\begin{array}{l}51(83.61 \%) \\
10(16.39 \%) \\
\end{array}$ & 0.0575 & 0.810 \\
\hline $\begin{array}{l}\text { Drinking } \\
\text { Yes } \\
\text { No }\end{array}$ & $\begin{array}{l}49(80.33 \%) \\
12(19.67 \%)\end{array}$ & $\begin{array}{l}48(78.69 \%) \\
13(21.31 \%)\end{array}$ & 0.0503 & 0.823 \\
\hline $\begin{array}{l}\text { Residence } \\
\text { Urban area } \\
\text { Rural area }\end{array}$ & $\begin{array}{l}42(68.85 \%) \\
19(31.15 \%)\end{array}$ & $\begin{array}{l}40(65.57 \%) \\
21(34.43 \%)\end{array}$ & 0.1488 & 0.700 \\
\hline
\end{tabular}

2.6. Statistical Treatment. The data obtained in this study were statistically analyzed and processed by SPSS20.0 software. Measurement data were expressed by $(\bar{x} \pm s)$ and tested by $t$-test. Enumeration data were expressed as $[n(\%)]$ and tested by $X^{2}$ test. The differences had a statistical significance when $P<0.05$.

\section{Results}

3.1. Comparison of the SAS and SDS Scores between the Two Groups. The SAS and SDS scores of patients at 24 hours after admission, 24 hours before the surgery, and 24 hours before discharge were compared and analyzed, and the results were as follows.

There were no significant differences in SAS and SDS scores of patients between the two groups at 24 hours after admission, as shown in Figure 1.

The SAS and SDS scores of the experimental group at 24 hours before the surgery were significantly better than those of the control group, as shown in Figure 2.

The SAS and SDS scores of the experimental group at 24 hours before discharge were significantly better than those of the control group, as shown in Figure 3.

3.2. Comparison of the Basic Conditions between the Two Groups in the Perioperative Period. According to the comparison between the basic conditions of the two groups in perioperative period, it is concluded that the length of hospitalization, length of catheter retention, and incidence of complications in the experimental group were significantly better than those in the control group $(P<0.05)$, with statistically significant differences, as shown in Table 2 .

\subsection{Comparison of the Nursing Satisfaction between the Two} Groups. The nursing satisfaction of the elderly patients with prostate cancer in the two groups was statistically

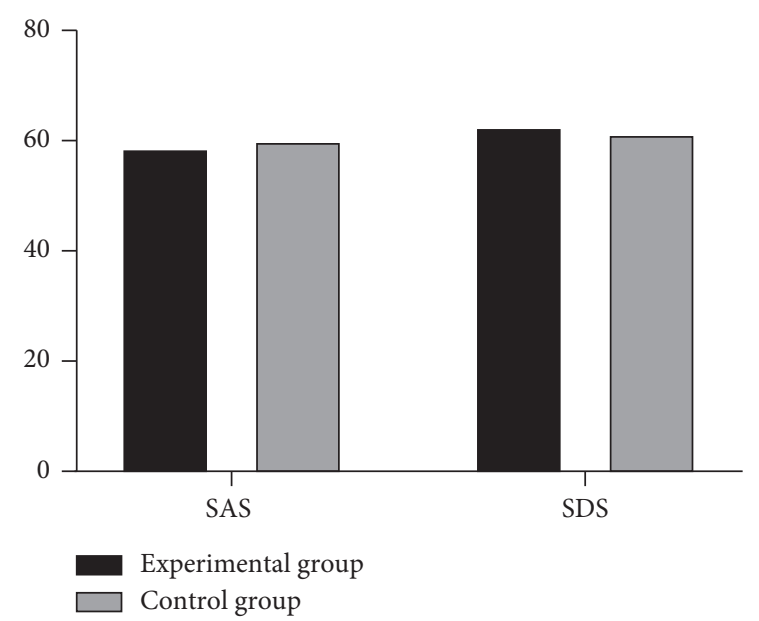

FIgURE 1: Comparison of SAS and SDS scores between the two groups at 24 hours after admission $(n=61)$. The abscissa represents SAS and SDS, while the ordinate represents score. The SAS and SDS scores in the experimental group at 24 hours after admission were $58.72 \pm 6.08$ and $62.56 \pm 7.91$, respectively. The SAS and SDS scores in the control group at 24 hours after admission were $60.02 \pm 6.32$ and $61.31 \pm 8.02$, respectively. There were no significant differences in SAS and SDS scores between the two groups at 24 hours after admission $(t=1.7578, P=0.2493)$.

analyzed in the form of questionnaires. According to the results, the total satisfaction of nursing in the experimental group was significantly higher than that in the control group $(P<0.05)$, with statistically significant differences, as shown in Table 3.

3.4. Comparison of the Quality of Life between the Two Groups after Surgery. The quality of life of the two groups was investigated and evaluated at three months after surgery, as shown in Figure 4. 


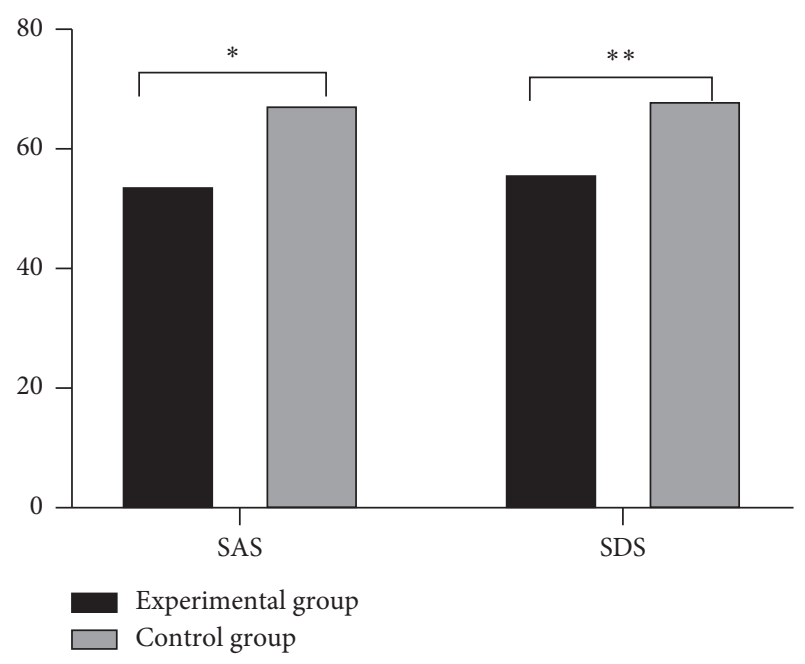

FIGURE 2: Comparison of SAS and SDS scores between the two groups at 24 hours before surgery $(n=61)$. The abscissa represents SAS and SDS, while the ordinate represents score. The SAS and SDS scores of the experimental group at 24 hours before surgery were $54.11 \pm 5.92$ and $56.12 \pm 6.46$, respectively. The SAS and SDS scores of the control group at 24 hours before surgery were $67.63 \pm 8.67$ and $68.37 \pm 7.13$, respectively. ${ }^{*}$ indicates that the SAS score of the experimental group was significantly better than that of the control group $(t=10.0582, P \leq 0.001) .{ }^{* *}$ indicates that the SDS score of the experimental group was significantly better than that of the control group $(t=9.9442, P \leq 0.001)$.

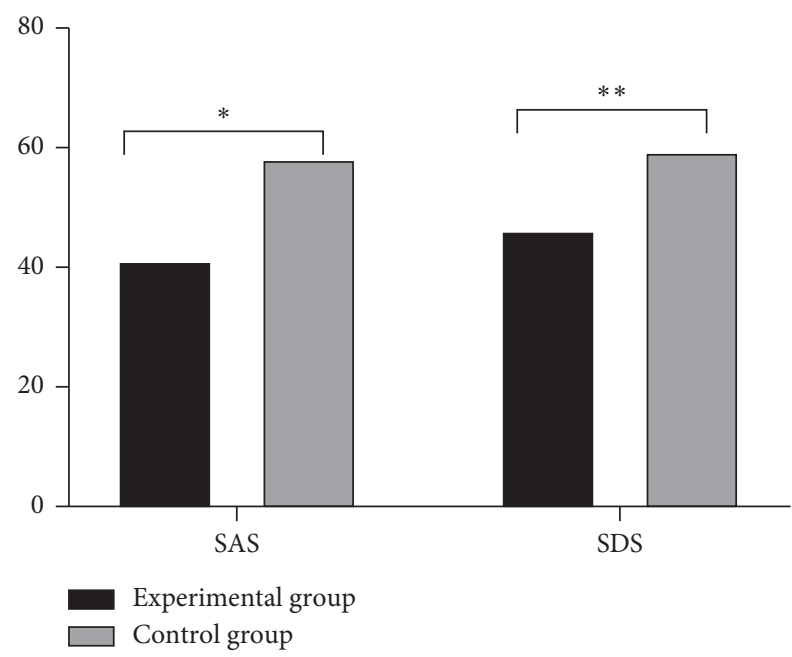

Figure 3: Comparison of SAS and SDS scores between the two groups at 24 hours before discharge $(n=61)$. The abscissa represents SAS and SDS, while the ordinate represents score. The SAS and SDS scores of the experimental group at 24 hours before discharge were $41.26 \pm 5.72$ and $46.31 \pm 6.01$, respectively. The SAS and SDS scores of the control group at 24 hours before discharge were $58.33 \pm 6.08$ and $59.48 \pm 7.11$, respectively. *indicates that the SAS score of the experimental group was significantly better than that of the control group $(t=15.9709, P \leq 0.001)$. ${ }^{* *}$ indicates that the SDS score in the experimental group was significantly better than that in the control group $(t=11.0487, P \leq 0.001)$.

\section{Discussion}

Prostate cancer is actually a relatively slow-growing tumor, and thus, patients may not be aware of the early-stage tumor growth. However, for patients with advanced cancer, the deterioration of the disease not only seriously impairs patients' physical function but also adversely affects patients' psychological states and quality of life [14-16]. With the development and progress of modern medical technology, the cure rate of the disease is increasing, and most patients with prostate cancer will receive compound kushen injection for comprehensive endocrine treatment in order to control the number of tumor cells, improve clinical symptoms, enhance the quality of life, and prolongsurvival time. However, the psychological changes of patients are often ignored in practice, and adverse psychological emotions or inadequate family support will also have a negative impact on the treatment effect of patients, leading to unsatisfactory results of compound kushen injection [17-20]. With the advancement of medicine, more attention has been paid to the psychological recovery of patients, especially in elderly patients with prostate cancer. Because the treatment method mainly targets the patients' special parts through the urinary system surgery, patients are prone to greater psychological burdens. Therefore, a scientific psychological intervention mode is more conducive to the postoperative rehabilitation of patients [21-24].

In this study, it was found that the evaluation of the psychological states of the experimental group was significantly better than that of the control group at 24 hours before surgery and 24 hours before discharge. On comparing the basic situations between the two groups during the perioperative period, the length of hospitalization, length of catheter retention after surgery, and incidence of complications in the experimental group were significantly better than those in the control group. According to the satisfaction in the two groups evaluated by the questionnaires, the satisfaction of the patients with the nursing process showed that the total satisfaction of nursing in the experimental group was significantly higher than that in the control group, and the quality of life score in the experimental group at three months after discharge was also significantly better than that in the control group. From the study results, it can be easily concluded that, due to the specificity of the surgery, the scores of preoperative psychological states in the two groups are high. When facing the upcoming surgery, the patients are easily restless, anxious, and stressed. However, the evaluation of patients' psychological states in the experimental group with the preoperative psychological intervention was significantly better than that in the control group, indicating that the psychological intervention combined with family cooperation can better help patients with good psychological construction, reduce the psychological stress of patients, and promote the postoperative rehabilitation of elderly patients with prostate cancer. The results of this study were similar to those of Beardo et al. [25], whose study showed that elderly patients with cancer were more 
TABLE 2: Comparison of the basic conditions between the two groups in the perioperative period $(n=61, \bar{x} \pm s)$.

\begin{tabular}{lccccc}
\hline Group & $\begin{array}{c}\text { Length of } \\
\text { operation }(\mathrm{min})\end{array}$ & $\begin{array}{c}\text { Bladder } \\
\text { irrigation time }(\mathrm{h})\end{array}$ & $\begin{array}{c}\text { Length } \\
\text { of hospitalization }(\mathrm{d})\end{array}$ & $\begin{array}{c}\text { Length of catheter } \\
\text { retention after surgery (d) }\end{array}$ & Incidence of complications \\
\hline Experimental group & $60.33 \pm 12.47$ & $23.56 \pm 7.04$ & $3.77 \pm 0.86$ & $2.74 \pm 0.78$ & $18.03 \%(11 / 61)$ \\
Control group & $59.62 \pm 12.74$ & $22.71 \pm 7.51$ & $5.71 \pm 1.06$ & $3.90 \pm 1.21$ & $34.43 \%(21 / 61)$ \\
$t / X^{2}$ & 0.3111 & 0.6449 & 11.1004 & 6.2933 & 4.2361 \\
$P$ & 0.7563 & 0.5202 & $P \leq 0.001$ & $P \leq 0.001$ & 0.040 \\
\hline
\end{tabular}

TABLE 3: Comparison of the nursing satisfaction between the two groups $(n=61)$.

\begin{tabular}{|c|c|c|c|c|}
\hline Group & Unsatisfied & Basically satisfied & Very satisfied & Total satisfaction \\
\hline Experimental group & $4.92 \%(3 / 61)$ & $29.51 \%(18 / 61)$ & $65.57 \%(40 / 61)$ & $95.08 \%(58 / 61)$ \\
\hline Control group & $22.95 \%(14 / 61)$ & $37.7 \%(23 / 61)$ & $39.34 \%(24 / 61)$ & $77.05 \%(47 / 61)$ \\
\hline$X^{2}$ & & & & 8.2700 \\
\hline$P$ & & & & 0.004 \\
\hline
\end{tabular}

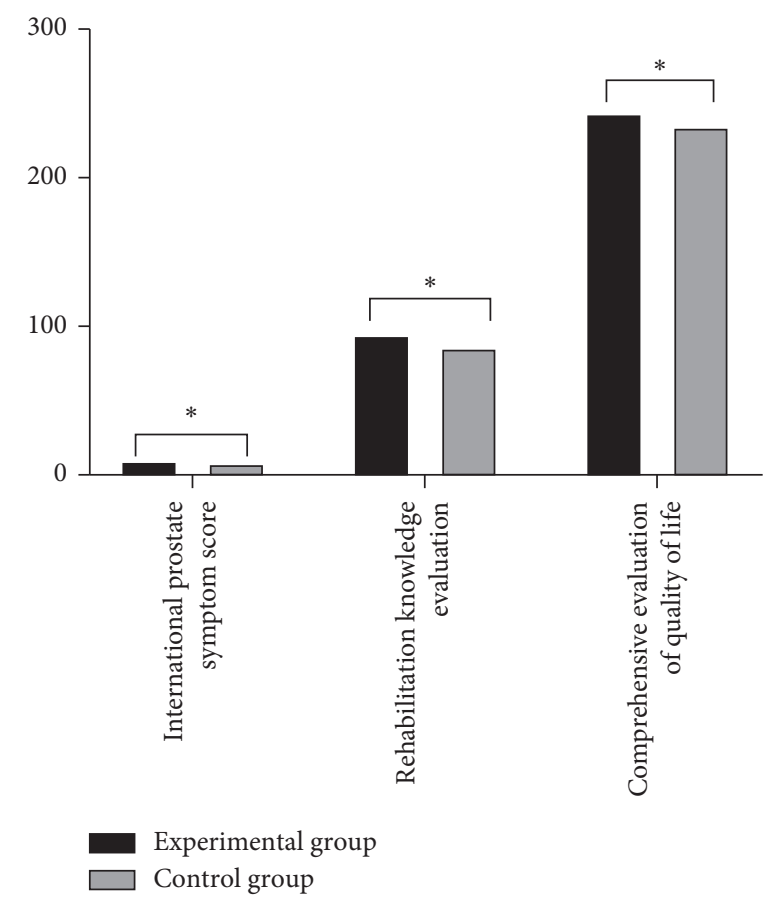

Figure 4: Comparison of the postoperative quality of life scores between the two groups $(n=61, \bar{x} \pm s)$. The abscissa represents international prostate symptom score, rehabilitation knowledge evaluation, and comprehensive evaluation of quality of life, while the ordinate represents score. The international prostate symptom score, rehabilitation knowledge evaluation, and comprehensive evaluation of quality of life in the experimental group were $9.67 \pm 3.71,94.47 \pm 3.12$, and $243.51 \pm 9.76$, respectively. The international prostate symptom score, rehabilitation knowledge evaluation, and comprehensive evaluation of quality of life in the control group were 8.21 $\pm 3.02,85.98 \pm 3.01$, and 234.72 \pm 10.49 , respectively. ${ }^{*}$ indicates that there were significant differences in the international prostate symptom score, rehabilitation knowledge evaluation, and comprehensive evaluation of quality of life between the two groups $(t=2.3837,15.2953,4.7914, P<0.05)$.

prone to emotional fluctuations, and patients had a better therapeutic effect under the supervision of nursing staff during hospital treatment. It also pointed out that many elderly patients after discharge were subject to decreased medication compliance due to the various effects of mood and life, thus leading to the occurrence of adverse symptoms, slow recovery of physical health, or treatment failure in severe cases. However, perioperative psychological intervention can effectively improve the patients' psychological states and enhance their treatment confidence.

In conclusion, psychological intervention combined with family support and adequate nursing care in the perioperative period for elderly patients with prostate cancer is more conducive to enhancing the patients' confidence in overcoming the disease. Both family support and care from the nursing staff can bring more psychological support to the 
elderly patients, which is easier for the patients to accept their conditions and improve their life quality, especially in the recovery process after surgery.

\section{Data Availability}

All primary data are available from the corresponding author upon a reasonable request.

\section{Conflicts of Interest}

The authors declare that there are no conflicts of interest.

\section{Authors' Contributions}

Jingyun Zhang and Caijian Li contributed equally to this article.

\section{References}

[1] C. C. M. van Laake-Geelen, R. J. E. M. Smeets, S. P. A. B. Quadflieg, J. Kleijnen, and J. A. Verbunt, "The effect of exercise therapy combined with psychological therapy on physical activity and quality of life in patients with painful diabetic neuropathy: a systematic review," Scandinavian Journal of Pain, vol. 19, no. 3, pp. 433-439, 2019.

[2] J. I. Steinert, L. D. Cluver, F. Meinck, D. Nzima, and J. Doubt, "Opening the black box: a mixed-methods investigation of social and psychological mechanisms underlying changes in financial behaviour," The Journal of Development Studies, vol. 56, no. 12, pp. 2327-2348, 2020.

[3] K. Whittingham, M. R. Sanders, L. McKinlay, and R. N. Boyd, "Parenting intervention combined with acceptance and commitment therapy: a trial with families of children with cerebral palsy," Journal of Pediatric Psychology, vol. 41, no. 5, pp. 531-542, 2016.

[4] M. Schmitter-Edgecombe, D. G. Dyck, and G. Dennis, "Cognitive rehabilitation multi-family group intervention for individuals with mild cognitive impairment and their carepartners," Journal of the International Neuropsychological Society, vol. 20, no. 9, pp. 897-908, 2014.

[5] T. P. Beauchaine, M. J. Reid, and C. Webster-Stratton, "Oneyear follow-up of combined parent and child intervention for young children with ADHD," Journal of Clinical Child and Adolescent Psychology, vol. 42, no. 2, pp. 251-261, 2013.

[6] B. Sun, L. Liu, X. Ren, and Z. Wang, "Psychological state of patients with sudden deafness and the effect of psychological intervention on recovery," Journal of International Medical Research, vol. 48, no. 9, 2020.

[7] Y. Tan, O. Gajic, P. J. Schulte, M. M. Clark, K. L. Philbrick, and L. V. Karnatovskaia, "Feasibility of a behavioral intervention to reduce psychological distress in mechanically ventilated patients," International Journal of Clinical and Experimental Hypnosis, vol. 68, no. 4, pp. 419-432, 2020.

[8] S. M. Powell, K. S. Fasczewski, D. L. Gill, and P. G. Davis, "Go with the FLOW: implementation of a psychological skills intervention in an exercise program for post-bariatric surgery patients," Journal of Health Psychology, vol. 25, no. 13-14, pp. 2260-2271, 2020.

[9] E. L. Addington, E. O. Cheung, and J. T. Moskowitz, "Who is most likely to benefit from a positive psychological intervention? moderator analyses from a randomized trial in people newly diagnosed with HIV," The Journal of Positive Psychology, vol. 15, no. 5, pp. 605-612, 2020.

[10] S. J. Heintzelman and K. Kushlev, "Emphasizing scientific rigor in the development, testing, and implementation of positive psychological interventions," The Journal of Positive Psychology, vol. 15, no. 5, pp. 685-690, 2020.

[11] R. L. Owens and L. Waters, "What does positive psychology tell us about early intervention and prevention with children and adolescents? a review of positive psychological interventions with young people," The Journal of Positive Psychology, vol. 15, no. 5, pp. 588-597, 2020.

[12] L. Stephenson, B. Hale, "An exploration into effectiveness of existential-phenomenological therapy as a UK NHS psychological treatment intervention," Journal of Humanistic Psychology, vol. 60, no. 3, pp. 436-453, 2020.

[13] J. L. Williams and A. A. Rheingold, "Novel application of skills for psychological recovery as an early intervention for violent loss: rationale and case examples," OMEGA-Journal of Death and Dying, vol. 81, no. 2, pp. 179-196, 2020.

[14] A. Heredia, F. Padilla, J. A. Castilla, and R. Garcia-Retamero, "Effectiveness of a psychological intervention focused on stress management for women prior to IVF," Journal of Reproductive and Infant Psychology, vol. 38, no. 2, pp. 113126, 2020.

[15] M. Tennant, J. McGillivray, G. J. Youssef, M. C. McCarthy, and T.-J. Clark, "Feasibility, acceptability, and clinical implementation of an immersive virtual reality intervention to address psychological well-being in children and adolescents with cancer," Journal of Pediatric Oncology Nursing, vol. 37, no. 4, pp. 265-277, 2020.

[16] M. Rusu, "Psychological optimization for adolescents with physical motor deficiency-a model of intervention," Psychology, vol. 11, no. 1, pp. 30-48, 2020.

[17] C. Amadi and B. M. Aleme, "The prevalence of zinc deficiency among men with and without prostate cancer in port harcourt, Nigeria," Nutrition and Cancer, vol. 72, no. 6, pp. 1018-1025, 2020.

[18] S. Löffeler, A. Halland, H. Fawad, C. Beisland, and E. S. Haug, "Non-metastatic prostate cancer: rationale for conservative treatment and impact on disease-related morbidity and mortality in the elderly," Scandinavian Journal of Urology, vol. 54, no. 2, pp. 105-109, 2020.

[19] M. Capece, M. Creta, A. Calogero et al., "Does physical activity regulate prostate carcinogenesis and prostate cancer outcomes? a narrative review," International Journal of Environmental Research and Public Health, vol. 17, no. 4, p. 1441, 2020.

[20] X. Cheng, Z.-H. Wang, M. Peng et al., "The role of radical prostatectomy and definitive external beam radiotherapy in combined treatment for high-risk prostate cancer: a systematic review and meta-analysis," Asian Journal of Andrology, vol. 22, no. 4, pp. 383-389, 2020.

[21] M. Prentice, A. Lewis, H. Payne, R. Davda, and M. Kebreya, "Radiotherapy in elderly patients with prostate cancer," Trends in Urology \& Men's Health, vol. 9, no. 4, pp. 13-17, 2018.

[22] M. Prentice, R. Davda, A. Nuhoglu Savas, and H. Payne, "Toxicity outcomes in the elderly prostate cancer patient," Clinical Oncology, vol. 30, no. 1, p. e37, 2018.

[23] P. Castellucci, C. Nanni, and V. Ambrosini, "Nuclear medicine imaging of prostate cancer in the elderly," Seminars in Nuclear Medicine, vol. 48, no. 6, pp. 541-547, 2018. 
[24] K. Jin, S. Qiu, J. Li et al., "How to choose proper local treatment in men aged $\geq 75$ years with cT2 localized prostate cancer?” Cancer Medicine, vol. 8, no. 7, pp. 3370-3378, 2019.

[25] P. Beardo, I. Osman, B. San José et al., "Safety and outcomes of new generation hormone-therapy in elderly chemotherapynaive metastatic castration-resistant prostate cancer patients in the real world," Archives of Gerontology and Geriatrics, vol. 82, pp. 179-185, 2019. 